\title{
TRAPPED BOSE-EINSTEIN CONDENSATES AT FINITE TEMPERATURE: A TWO-GAS MODEL
}

\author{
R.J. DODD \\ Institute for Physical Science and Technology \\ University of Maryland at College Park \\ College Park, MD 20742, USA \\ K. BURNETT \\ Clarendon Laboratory, Department of Physics, University of Oxford \\ Parks Road, Oxford OX1 3PU, UK
}

M. EDWARDS

Department of Physics, Georgia Southern University, Statesboro, GA 30460, USA

and Ch.W. Clark

Electron and Optical Physics Division

National Institute of Standards and Technology

Gaithersburg, MD 20899, USA

A simple picture describes the results of recent treatments of partially-condensed, dilute, trapped Bose gases at temperature $T>0$. The condensate wa ve function is nearly identical to that of a $T=0$ condensate with the same number of condensate atoms, $N_{0}$. The cloud of non-condensed atoms is described by the statistical mechanics of an ideal Bose gas in the combined potentials of the magnetic trap and the cloud-condensate interaction. We provide a physical motivation for this result, show how it emerges in the Hartree-Fock-Bogoliubov-Popov approximation, and explore some of its implications for future experiments.

PACS numbers: 03.75.Fi, 67.40.Db, 67.90.+z

\section{Introduction}

Most recent experiments [1-3] on dilute, magnetically-trapped, alkali-atom Bose gases have viewed phenomena which are well described by the zero-temperature $(T=0)$ mean-field theory of the Bose-Einstein condensate (BEC), in which virtually all the gas in the system resides in the condensed state. The technique of evaporative cooling [4] used in all such experiments, grows the BEC by selective 
extraction of the non-condensed, "thermal" component of the gas, which is located at the outer edges of the trap [5]. The $T=0$ mean-field theory has been found to give a good account of many BEC properties observed in these systems [6,7]. New experiments $[8,9]$ have started to probe BECs at $T>0$, and so the testing of alternative finite-temperature BEC theories has begun.

This paper draws attention to common features emerging from several independent finite-temperature theories, which suggest that a relatively simple picture, which we call the "two-gas model", describes many of the properties of a dilute Bose gas with repulsive atomic pair interactions (scattering length $a>0$ ). The two gases concerned are the condensate gas, the intrinsic properties of which are essentially independent of temperature; and the thermal, non-condensed gas, which behaves much like an ideal Bose gas at temperature $T$ in an effective potential created by the condensate. This model emerges naturally as a limiting case of the Hartree-Fock-Bogoliubov-Popov (HFB-Popov) [10-12] and Hartree-Fock (HF) [13] approximations, but its features seem also to be manifested in recent quantum Monte Carlo [14] and semiclassical [15] calculations. The two-gas picture offers some straightforward implications for interpretation of experiments and for further development of first-principles theories.

Our evidence for the validity of this picture first emerged from large-scale numerical calculations, but its origin can be traced back qualitatively within the structure of finite-temperature field theory. In Sec. 2, we show how such a theory can plausibly lead to a two-gas scenario. Section 3 explores some of the implications of the model.

\section{Two-gas model as a limit of the HFB-Popov approximation}

The HFB-Popov equations have been derived elsewhere [10] and we merely state the basic equations here. In the Heisenberg equation of motion, the Bose field operator, $\widehat{\psi}(r)$ is decomposed into a $c$-number wave function $\psi_{0}(r)$ that describes a condensate of $N_{0}$ atoms, and an operator $\widetilde{\psi}(\boldsymbol{r})$ describing the non-condensate atoms: $\widehat{\psi}(\boldsymbol{r})=\sqrt{N_{0}} \psi_{0}(\boldsymbol{r})+\widetilde{\psi}(\boldsymbol{r})$. In the HFB-Popov approximation, the wave function for a condensate of trapped atoms satisfies a generalized Gross-Pitaevskii (GP) equation

$$
\left\{\widehat{H}_{0}+U_{0}\left[N_{0}\left|\psi_{0}(\boldsymbol{r})\right|^{2}+2 \widetilde{n}(\boldsymbol{r})\right]\right\} \psi_{0}(\boldsymbol{r})=\mu \psi_{0}(\boldsymbol{r}),
$$

where $\widehat{H}_{0}=\frac{-\hbar^{2}}{2 M} \nabla^{2}+V_{\text {trap }}(r)$ is the Hamiltonian for a single atom of mass $M$ and position coordinate $r$; the trapping potential (for cylindrically symmetric systems of current interest) is given by $V_{\text {trap }}(r)=M\left(\omega_{\rho}^{2} \rho^{2}+\omega_{z}^{2} z^{2}\right) / 2$, with $\omega_{\rho}$ and $\omega_{z}$ the radial and axial angular frequencies of the trap; $U_{0}=4 \pi \hbar^{2} a / M$ expresses the binary interaction between atoms; the chemical potential $\mu$, interpreted as the work required to add one more atom to the condensate, is treated as an eigenvalue; and $\psi_{0}(r)$ is normalized to unity.

The function $\tilde{n}(\boldsymbol{r})$ is the density of the non-condensed component of the gas,

$$
\widetilde{n}(\boldsymbol{r})=\sum_{j}\left\{\left[\left|u_{j}(\boldsymbol{r})\right|^{2}+\left|v_{j}(\boldsymbol{r})\right|^{2}\right] N_{j}+\left|v_{j}(\boldsymbol{r})\right|^{2}\right\}
$$


where

$$
N_{j}=\left[\exp \left(\beta E_{j}\right)-1\right]^{-1}
$$

is the Bose-Einstein factor, $\beta^{-1}=k_{\mathrm{B}} T$ and $k_{\mathrm{B}}$ is the Boltzmann constant. The quasi-particle excitation energies $E_{j}$ and amplitudes $u_{j}(r), v_{j}(r)$ are obtained by solution of the coupled HFB-Popov equations

$$
\begin{aligned}
& \widehat{\mathcal{L}} u_{j}(\boldsymbol{r})+N_{0} U_{0}\left|\psi_{0}(\boldsymbol{r})\right|^{2} v_{j}(\boldsymbol{r})=E_{j} u_{j}(\boldsymbol{r}), \\
& \widehat{\mathcal{L}} v_{j}(\boldsymbol{r})+N_{0} U_{0}\left|\psi_{0}(\boldsymbol{r})\right|^{2} u_{j}(\boldsymbol{r})=-E_{j} v_{j}(\boldsymbol{r}),
\end{aligned}
$$

where $\widehat{\mathcal{L}} \equiv \widehat{H}_{0}+2 U_{0} n(r)-\mu$, and $n(r)=N_{0}\left|\psi_{0}(r)\right|^{2}+\widetilde{n}(r)$ is the total trapped-atom density.

In simple physical terms, Eqs. (1)-(5) describe a condensate subject to interaction with itself and a thermal cloud, with the cloud being generated by thermal excitations of condensate quasi-particles. (There is also a non-thermal contribution to this cloud, the so-called "quantum depletion" term represented by the rightmost term of Eq. (2), but it is much smaller than the thermal component except near $T=0$.) To solve these equations for a given atomic species and trap configuration, we fix the values of $T$ and $N_{0}$, and then determine all other quantities self-consistently, eventually obtaining the total number of trapped atoms, $N$, via

$$
N=\int \mathrm{d} \boldsymbol{r} n(\boldsymbol{r})=N_{0}+\sum_{j} N_{j} .
$$

By carrying out a sequence of such (laborious) calculations, we can map out the $\left\{N, N_{0}, T\right\}$ phase diagram of the interacting Bose gas. We present elsewhere [12] a detailed comparison of the results of this approach with experimental data for the ${ }^{87} \mathrm{Rb}$ condensate at JILA [9]; for the temperature-dependent quasiparticle excitation energies, HFB-Popov approximation agrees with experiment to within $5 \%$ for temperatures from zero up to $65 \%$ of the temperature $T_{0}$ of the phase transition for the corresponding ideal gas (corresponding to thermal fractions from less than $1 \%$ to about $50 \%$ ). Although at present there are considerable discrepancies as $T \rightarrow T_{0}$, it seems that HFB-Popov approximation is a useful working theory over a significant temperature range.

Several calculations $[11,12]$ have shown that, for current experiments, the quantum depletion of a small condensate is negligible, i.e., $\left.\int \mathrm{d} \boldsymbol{r} \tilde{n}(\boldsymbol{r})\right|_{T=0}=$ $\sum_{j} \int \mathrm{d} \boldsymbol{r}\left|v_{j}(\boldsymbol{r})\right|^{2} \ll N_{0}$. This justifies use of the approximation

$$
v_{j}(r) \equiv 0 \text {, }
$$

which is equivalent to the Hartree-Fock approximation used by other authors $[13,16]$. If we apply this approximation to Eq. (4) (and neglect the contribution of $\tilde{n}(r)$ to $n(r))$, we obtain an ordinary Schrödinger equation for $u_{j}(r)$,

$$
\left[-\frac{\hbar^{2}}{2 M} \nabla^{2}+V_{\mathrm{eff}}(r)\right] u_{j}(r)=E_{j} u_{j}(r)
$$

where the effective potential, given by

$$
V_{\text {eff }}(r)=V_{\text {trap }}(r)+2 N_{0} U_{0}\left|\psi_{0}(r)\right|^{2}
$$

is that of the trap modified by the repulsive pair interaction between the thermally-excited atoms and the condensate density. 
If we consider the case of a relatively small thermal fraction, then we expect to find the condensate localized near the center of the trap, so that Eq. (9) presents the thermal cloud with a trap and repulsive core. Thus, at least the low-energy quasi-particle amplitudes $u_{j}(r)$ will be expelled from the core, i.e., they will have little overlap with the condensate wave function. This then gives consistency of Eq. (5) with our initial approximation, Eq. (7). It also justifies the approximation that completes our portrayal of a two-gas system: because of the expulsion of quasi-particle amplitudes from the condensate, we assume that $\widetilde{n}(\boldsymbol{r})$ can be neglected in Eq. (1), so that the condensate wave function is determined by solving

$$
\left[-\frac{\hbar^{2}}{2 M} \nabla^{2}+V_{\text {trap }}(r)+N_{0} U_{0}\left|\psi_{0}(r)\right|^{2}\right] \psi_{0}(r)=\mu \psi_{0}(r)
$$

which is just the usual GP equation for a condensate of $N_{0}$ atoms at $T=0$.

Thus, these arguments have led us to a simple picture in which the finite-temperature Bose system appears to be composed of two distinct gases. One of these gases, the BEC, is always effectively at zero temperature, and is described by an equation which depends only on its own atomic population, $N_{0}$, and the trap parameters. The other gas, the thermal cloud, behaves as a normal Bose gas at finite temperature, sensing the presence of the condensate through an elastic interaction; it does not undergo Bose-Einstein condensation itself, but serves as an atomic reservoir for the BEC. This resembles the phenomenon of BEC of an ideal gas in an external potential, except that we account for interactions in the identification of the ground state, and in the modification by the condensate of the external potential exposed to the thermal cloud.

\section{Implications of the two-gas model}

The two-gas model provides us with a straightforward way of computing the phase diagram of the dilute Bose gas for $T<T_{0}$. If there are $N_{0}$ atoms in the $\mathrm{BEC}$, we solve Eq. (10) to obtain what we will call an equivalent zero-temperature condensate (EZC), i.e. the corresponding $T=0$ condensate that contains $N_{0}$ atoms. The EZC solution provides us an orbital $\psi_{0}\left(r ; N_{0}\right)$ and chemical potential $\mu\left(N_{0}\right)$; with these in hand, we can construct $V_{\text {eff }}(r)$ and find the spectrum of Eq. (8). This procedure, which is independent of $T$, gives us the information we need to compute the equilibrium value of $N$ for given values of $N_{0}$ and $T$ : we evaluate Eq. (6) from Eq. (3).

In short, the EZC provides a "reference condensate" which, for a given set of trap parameters, describes all systems with the same number of condensate atoms $N_{0}$. As we have shown elsewhere [12], this model provides good agreement with the results of full HFB-Popov calculations of condensate and thermal densities and the critical temperature $T_{0}$; the emergence of an EZC can also be seen in the analysis by Krauth [14] of the results of his quantum Monte Carlo calculations, and in the recent quasi-classical calculations of Minguzzi et al. [15]. Comparison of the EZC condensate densities with those of HFB-Popov calculations is made in Fig. 1; this shows that even in cases where the condensate fraction $f=N_{0} / N$ is as small as 0.1 , the condensate is relatively unperturbed by the presence of the thermal cloud. 


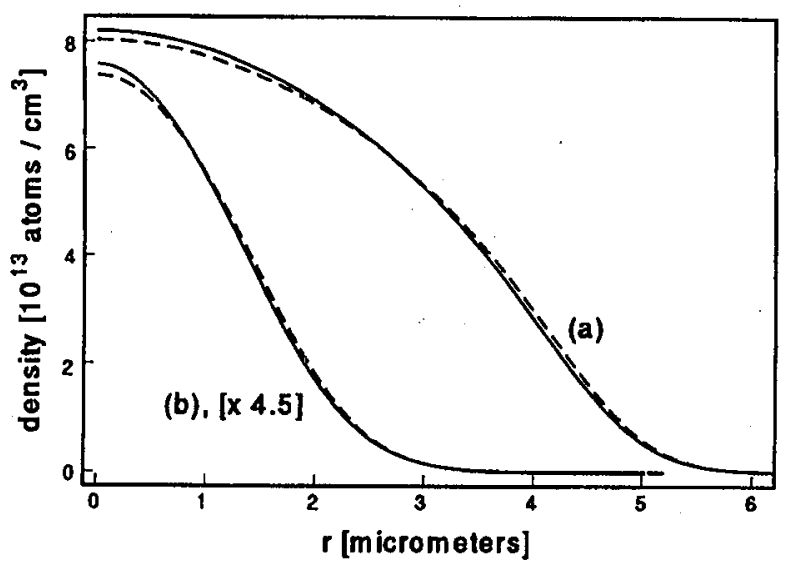

Fig. 1. Condensate densities in the $z=0$ plane for systems of ${ }^{87} \mathrm{Rb}$ atoms in the JILA TOP trap [1] with radial frequency $\nu_{\rho}=74 \mathrm{~Hz}$. The solid line shows the condensate density as computed in the HFB-Popov approximation, and the dashed line is the density of the corresponding EZC. Case (a): A system of $N=13150$ atoms at $T=70 \mathrm{nK}$, corresponding to $f=0.54$ in the HFB-Popov approximation, i.e. $N_{0}=7106$. (b) A system of $N=2000$ atoms at $T=51 \mathrm{nK}$, corresponding to $f=0.1$. The EZC densities are seen to be very close to those of the HFB-Popov approximation.

Another straightforward implication of the two-gas model concerns the density profile of the thermal cloud. If we entertain the simple hypothesis that the cloud would be described by classical statistical mechanics of an ideal gas, then its density $\tilde{n}(r)$ would be proportional to $\exp \left[-\beta V_{\text {eff }}(r)\right]$. Since $V_{\text {eff }}(r)$ is repulsive at small $|r|$ and confining at large $|r|, \widetilde{n}(r)$ will attain its maximum away from the center of the condensate. If we consider the Thomas-Fermi limit [17] appropriate to large condensates, then for the case of a spherical condensate $\left[V_{\text {trap }}(r)=M \omega^{2} r^{2} / 2\right]$ we find that

$$
V_{\mathrm{eff}}(\boldsymbol{r})= \begin{cases}\left(M \omega^{2} / 2\right)\left(2 r_{\mathrm{TF}}^{2}-r^{2}\right), & r<r_{\mathrm{TF}}, \\ M \omega^{2} r^{2} / 2, & r>r_{\mathrm{TF}},\end{cases}
$$

where the Thomas-Fermi radius, $r_{\mathrm{TF}}=\left(\frac{15 N_{0} U_{0}}{4 \pi M \omega^{2}}\right)^{1 / 5}$, defines the sharp boundary of the condensate. Thus in this limit, $\tilde{n}(r)$ is largest at the surface of the condensate, and its distribution becomes more localized as $N_{0}$ increases, albeit slowly.

The key qualitative aspects of this classical description are applicable to the quantum system, as shown in Fig. 2: this displays results of a full quantum-mechanical finite-number description, without any of the semiclassical continuous spectrum approximations made by other authors $[15,18]$. This figure clearly suggests that quantitative interpretations of experimental data on finite-temperature condensates, e.g. determination of a condensate fraction from density measurements, will have to invoke some detailed model of the thermal distribution, since this distribution is neither monotonic nor close to the results obtained for a noninteracting gas. On the other hand, our current model suggests that the condensate and thermal densities are relatively distinctly segregated within the cloud, 


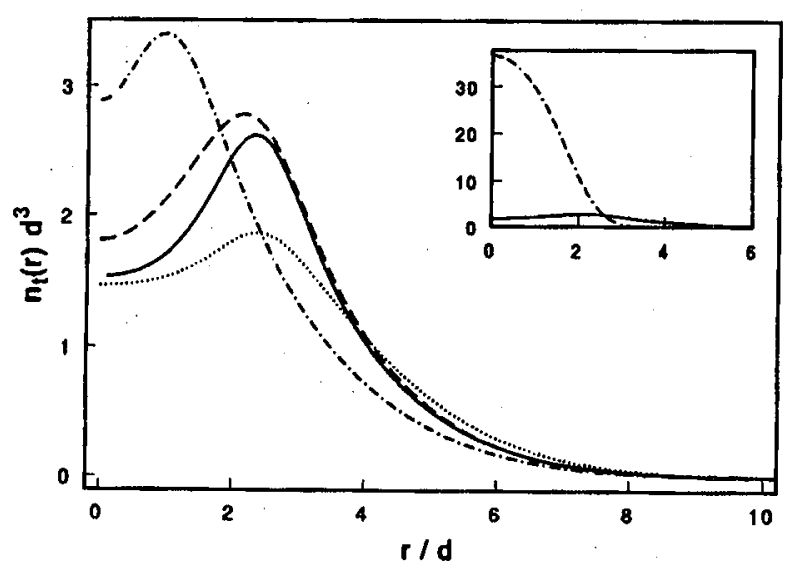

Fig. 2. Thermal density for $N \approx 2000{ }^{87} \mathrm{Rb}$ atoms in a spherical trap with $\nu_{r}=\omega / 2 \pi=$ $200 \mathrm{~Hz}, T=75 \mathrm{nK}$, corresponding to $f=0.5$ in the HFB-Popov approximation. The radius $r$ is given in units of the characteristic length of the oscillator $d=\sqrt{\hbar / M \omega}$. These parameters were previously used by Hutchinson et al. [11], whose numerical HFB-Popov results we have reproduced and use here. The main figure shows the thermal density as computed in various approximations. Chain-dashed line: the confined ideal quantum gas; dashed line: full solution of the HFB-Popov equations; solid line: full quantum-statistical distribution as computed from two-gas model, which obtains $N=1965$ (vs. the exact value of 2000) from $N_{0}=1000$ and $T=75 \mathrm{nK}$; dotted line: result of classical statistiral mechanics applied to the two-gas model, using a fit to force $N=2000$. The inset compares the HFB-Popov thermal density (solid line) with that of the condensate (dashed). Thus, even when the system is only $50 \%$ condensate, the peak condensate density is clearly much higher than that of the thermal cloud.

which may considerably simplify the qualitative understanding of some properties. Since it originates in the distinction between interactions of condensate and non-condensate atoms, this spatial segregation of the two gases seems to be a fundamental aspect of the behavior of inhomogeneous Bose gases, such as the trapped-atom systems of current interest. This may have interesting consequences for applications: for example, it may be possible to selectively extract condensate vs. thermal atoms from a trap by appropriate positioning of a probe, thus obtaining an outcoupled matter wave with higher coherence than would be otherwise expected [1.9]. In homogeneous systems, on the other hand, condensate and non-condensate populations are intertwinned; this is one of the essential features of the two-fluid model of liquid helium [20].

Taking this idea further, we suggest that any property of a finite-temperature BEC should be compared in the first instance to that of the corresponding EZC. In the two-gas model, we expect most of the $T$-dependence of a given quantity to be reduced to $N_{0}$-dependence. For example, in Fig. 3 we show the quasiparticle excitation frequencies for the JILA TOP trap, over a range of temperatures relevant to recent experiments, as computed in the full HFB-Popov approximation and in the 


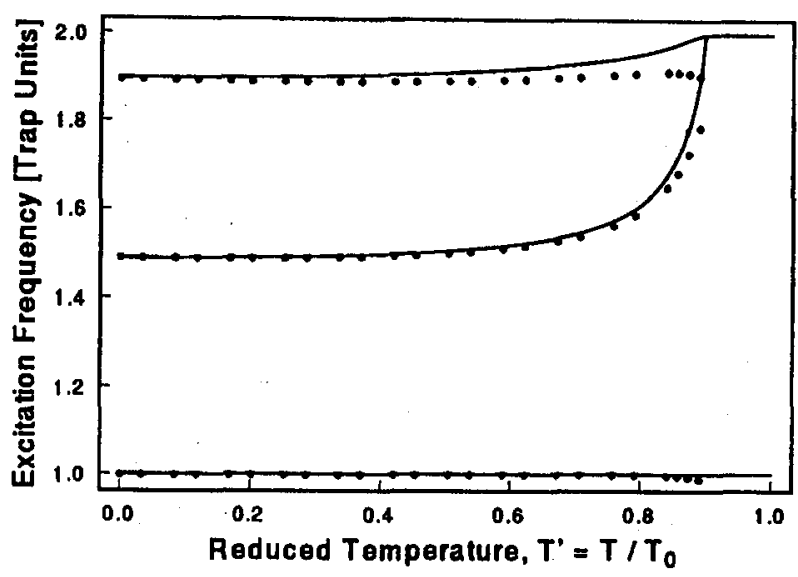

Fig. 3. HFB-Popov excitation frequencies (filled circles) for the $m=0$ (top), $m=2$ (middle), and the $m=1$ modes (bottom) for the JILA TOP trap with radial frequency $\nu_{r}=129 \mathrm{~Hz}$ and $N=2000{ }^{87} \mathrm{Rb}$ atoms, vs. temperature in units of $T_{0}$. Overlaid (solid lines) are the frequencies for a zero-temperature system with the same number $N_{0}$ of condensate atoms as in the finite-temperature system.

two-gas model. It is seen that the two methods agree up to temperatures quite close to the phase transition, so the main effect of finite temperature is renormalization of the value of $N_{0}$. An analogous result was seen in earlier calculations [21,22] for the homogeneous Bose-condensed gas of the temperature dependence of the speed of sound, which found it to be given by an equivalent $T=0$ expression adjusted for the temperature-dependence of the condensate density.

\section{Conclusions}

We find that condensate and thermal populations of a partially Bose-Einstein-condensed trapped-atom system separate out to a considerable extent. Treating the condensate as uncoupled from the thermal cloud, and the thermal cloud as interacting with a static condensate potential, yields results similar to those that come from involved self-consistent field calculations. These results motivate the identification of the equivalent zero-temperature condensate as a consolidating feature of finite-temperature systems. In this model, the main effect of finite temperature on the condensate is depletion of the condensate number. Condensate properties that depend only weakly upon $N_{0}$, such as the quasi-particle spectrum in the large- $N_{0}$ limit (corresponding to the excitation frequencies of large condensates) [7], should exhibit only weak temperature dependence.

\section{Acknowledgments}

This work was supported in part by the U.S. National Science Foundation under grants PHY-9601261 and PHY-9612728, the U.S. Office of Naval Research, by MCS grant No. PAN/NIST-93-156, and by the U.K. Engineering and Physical Sciences Research Council. 


\section{References}

[1] M.H. Anderson, J.R. Ensher, M.R. Matthews, C.E. Wieman, E.A. Cornell, Science 269, 198 (1995); C.J. Myatt, E.A. Burt, R.W. Ghrist, E.A. Cornell, C.E. Wieman, Phys. Rev. Lett. 78, 586 (1997).

[2] K.B. Davis, M.-O. Mewes, M.R. Andrews, N.J. van Druten, D.S. Durfee, D.M. Kurn, W. Ketterle, Phys. Rev. Lett. 75, 3969 (1995); M.-O. Mewes, M.R. Andrews, N.J. van Druten, D.M. Kurn, D.S. Durfee, W. Ketterle, Phys. Rev. Lett. 77, 416 (1996); M.-O. Mewes, M.R. Andrews, N.J. van Druten, D.M. Kurn, D.S. Durfee, C.G. Townsend, W. Ketterle, Phys. Rev. Lett. 77, 988 (1996); M.-O. Mewes, M.R. Andrews, D.M. Kurn, D.S. Durfee, C.G. Townsend, W. Ketterle, Phys. Rev. Lett. 78, 582 (1997); M.R. Andrews, M.-O. Mewes, N.J. van Druten, D.S. Durfee, D.M. Kurn, W. Ketterle, Science 273, 84 (1996); M.R. Andrews, C.G. Townsend, H.-J. Miesner, D.S. Durfee, D.M. Kurn, W. Ketterle, Science 275, 637 (1997).

[3] C.C. Bradley, C.A. Sackett, J.J. Tollet, R.G. Hulet, Phys. Rev. Lett. 75, 1687 (1995); C.C. Bradley, C.A. Sackett, R.G. Hulet, Phys. Rev. Lett. 79, E1170 (1997); C.C. Bradley, C.A. Sackett, R.G. Hulet, Phys. Rev. Lett. 78, 985 (1997).

[4] W. Ketterle, N.J. van Druten, in: Advances in Atomic, Molecular, and Optical Physics, Vol. 37, Eds. B. Bederson, H. Walther, Academic, New York 1997.

[5] E. Cornell, J. Res. Nat. Inst. Stand. Technol. 101, 419 (1996).

[6] M. Holland, J. Ccoper, Phys. Rev. A 53, R1954 (1996); M. Edwards, R.J. Dodd, C.W. Clark, P.A. Ruprecht, K. Burnett, Phys. Rev. A 53, R1950 (1996); M. Edwards, P.A. Ruprecht, K. Burnett, R.J. Dodd, C.W. Clark, Phys. Rev. Lett. 77, 1671 (1996); M. Edwards, R.J. Dodd, C.W. Clark, K. Burnett, J. Res. Natl. Inst. Stand. Technol. 101, 553 (1996); R.J. Dodd, M. Edwards, C.J. Williams, C.W. Clark, M. Holland, P.A. Ruprecht, K. Burnett, Phys. Rev. A 54, 661 (1996); Y. Castin, R. Dum, Phys. Rev. Lett. 77, 5315 (1996); V.M. Perez-Garcia, H. Michinel, J.I. Cirac, M. Lewenstein, P. Zoller, Phys. Rev. Lett. 77, 5320 (1996); B.D. Esry, Phys. Rev. A 55, 1147 (1997); M.J. Holland, D.S. Jin, M.L. Ciofalo, J. Cooper, Phys. Rev. Lett. 78, 3801 (1997).

[7] S. Stringari, Phys. Rev. Lett. 77, 2360 (1996).

[8] J.R. Ensher, D.S. Jin, M.R. Andrews, C.E. Weiman, E.A. Cornell, Phys. Rev. Lett. 77, 4984 (1996).

[9] D.S. Jin, M.R. Matthews, J.R. Ensher, C.E. Wieman, E.A. Cornell, Phys. Rev. Lett. 78, 764 (1997).

[10] A. Griffin, Phys. Rev. B 53, 9341 (1996).

[11] D.A.W. Hutchinson, E. Zaremba, A. Griffin, Phys. Rev. Lett. 78, 1842 (1997).

[12] R.J. Dodd, M. Edwards, C.W. Clark, K. Burnett, Phys. Rev. A, in press; R.J. Dodd, K. Burnett, M. Edwards, C.W. Clark, submitted to Phys. Rev. A.

[13] T. Bergeman, Phys. Rev. A 55, 3658 (1997).

[14] W. Krauth, Phys. Rev. Lett. 77, 3695 (1996).

[15] A. Minguzzi, S. Conti, M.P. Tosi, J. Phys., Condens. Matter 9, L33 (1997).

[16] M. Houbiers, H.T.C. Stoof, Phys. Rev. A 55, 5055 (1996).

[17] G. Baym, C. Pethick, Phys. Rev. Lett. 76, 6 (1996).

[18] S. Giorgini, L.P. Pitaevskii, S. Stringari, Phys. Rev. Lett. 78, 3987 (1997). 
[19] R.J. Dodd, C.W. Clark, M. Edwards, K. Burnett, Optics Express 1, 284 (1997).

[20] As reviewed, for example, by R.P. Feynman, Statistical Mechanics, Addison Wesley, Reading, Massachusetts 1972, Ch. 11; A. Griffin, Excitations in a Bose-Condensed Liquid, Cambridge University Press, Cambridge 1993.

[21] P. Szépfalusy, I. Kondor, Ann. Phys. (N.Y.) 82, 1 (1974).

[22] S.H. Payne, A. Griffin, Phys. Rev. B 32, 7199 (1985). 